\title{
Environmental Modifications and Supports for Participation Among Adults Aging With Intellectual and Developmental Disabilities: A Scoping Review
}

\author{
Selena E. Washington, Khalilah R. Johnson, Jesse M. Hollenbeck
}

Importance: Adults aging with intellectual and developmental disabilities (IDD) continue to face barriers to participation in meaningful occupation; moreover, the increased life expectancy of adults with IDD intensifies the need for age- and environmentspecific support.

Objective: To map and categorize transdisciplinary literature on environmental modifications and supports for adults aging with IDD.

Data Sources: Studies published between January 1, 2000, and January 1, 2019, identified through PubMed and Scopus.

Study Selection and Data Collection: Eleven articles met the inclusion criteria and contained information specific to occupational participation based on environmental modification (e.g., physical space, technology, universal design, type of living setting), social supports (e.g., family members, caretakers, peer groups, health care professionals, policies, organizational infrastructure), or both and adults age $\geq 35$ yr with IDD.

Findings: Eleven studies met the criteria for this scoping review. Thematic coding was used to examine the articles within one or more themes: definition of need, environmental risk and assessment, environmental setting, intervention and program planning, use of technology, and bureaucracy.

Conclusions and Relevance: This review highlights collective and individual outcomes in the areas of assessment, intervention, and advocacy. Further research is needed within the scope of occupational therapy and disability studies that examines environmental factors and participation outcomes in this population.

What This Article Adds: The authors define the interconnectedness of adults aging with IDD and environmental factors, identify barriers to participation, and guide occupational therapy practitioners' logic on how to positively affect environmental change and supports through intervention.

$T^{2}$ he needs of older adults with intellectual and developmental disabilities (IDD) constitute a social phenomenon that poses significant and unexpected challenges. Key among those challenges will be the political and professional response to both the new needs that this population presents and the exacerbation of existing needs (Alcedo et al., 2017). Intellectual disability is a developmental disability that is diagnosed before age $18 \mathrm{yr}$ and is characterized by limitations in intellectual functioning (e.g., learning, reasoning, problem solving) and adaptive behavior (e.g., practical and social skills) that affect all aspects of daily life (American Association on Intellectual and Developmental Disabilities, n.d.). Although occupational therapy practitioners primarily work with children and youth with IDD, they are uniquely positioned to work with this population across the life course (Johnson et al., 2019). Improvements in primary health care and management, technology, and built and social environments have contributed to increased life expectancy for people with IDD (Miskimmin et al., 2019); consequently, practitioners and researchers interested in adults aging with IDD have identified areas of significant concern (Hole et al., 2013).

Citation: Washington, S. E., Johnson, K. R., \& Hollenbeck, J. M. (2021). Environmental modifications and supports for participation among adults aging with intellectual and developmental disabilities: A scoping review. American Journal of Occupational Therapy, 75, 7504180060. https://doi.org/ 10.5014/ajot.2021.045336 
Because people with neurodevelopmental and genetic disorders have a disposition toward poorer health and sedentary behaviors, people with IDD experience the aging process earlier and at an accelerated rate than those without IDD (Heller, 2017; Hole et al., 2013). Despite the implementation of programmatic supports for people with IDD, adults aging with IDD continue to face social and physical barriers to participation in meaningful occupations (Hammel et al., 2015; Johnson \& Bagatell, 2017; Johnson et al., 2019). Moreover, people with IDD have personal factors, such as an increased risk for age-related physiological changes and chronic illnesses, that can negatively influence their occupational participation with or without proper support (Hammel et al., 2015; Spassiani et al., 2019).

A large proportion of adults aging with IDD live in community-based or independent housing, chiefly because of the deinstitutionalization movement (Hutchings et al., 2008); however, the lack of support for occupational engagement in their communities is pervasive (Miskimmin et al., 2019). Scholars have acknowledged that adults with IDD have "merely become physically relocated into the community, rather than reintegrated as meaningfully engaged citizens" (Spassiani et al., 2019, p. 1465). This acknowledgment, coupled with an increased life expectancy, demonstrates a need for nuanced understanding of how aging influences the occupational participation of adults with IDD and intensifies the need for age- and environment-specific supports. Hammel et al. (2015) identified eight environmental factors that affect participation for this population: built, natural, assistive technology, access to information and technology, transportation, social support and societal attitudes, economics, and systems and policies. Despite the expansion of community-based services and initiatives aimed at ameliorating these barriers, adults aging with IDD continue to feel excluded and not valued as community members (Hammel et al., 2015; Spassiani et al., 2019).

Supports for adults aging with IDD have focused on expanding social networks, improving community participation and leisure, enhancing medical services (particularly primary care, especially as it relates to dementia care), and overall quality of life (Hammel et al., 2015; Schalock, 2004; Schepens et al., 2019). A review by Schepens et al. (2019) concentrated on identifying support strategies at the governmental, organizational, and individual levels that affect quality-of-life outcomes for adults aging with IDD; this scoping review contributes to these age-specific IDD support practices. We found many strategies for this population centered on aging in place, elder care with specialization in IDD, transition planning for those who were employed, lifestyle design, relationship building, access to preventive and general health care, health education, and enhanced government funding. These studies are informative regarding quality of life, and it is understood that interest in an occupation, tasks, or service precedes participation. However, interest may not lead to participation because of environmental barriers (Law, 2002).

Occupational therapy scholars have long challenged practitioners to reconsider aspects of practice by recognizing the power of the environment as a means of intervention (Kielhofner, 2005; Law, 1991, 2002), yet there remains a dearth of evidence specific to environmental modifications and supports that can inform occupational therapy practice with adults aging with IDD. In this scoping review, we map and categorize transdisciplinary literature demonstrating the impact of environmental and assistive technology modifications that meet the occupational needs of adults aging with IDD as well as the unique characteristics of supported and modified living settings to facilitate occupational participation.

\section{Disability Perspectives and the Environment}

The social model of disability (Oliver, 2004) and Law's (1991) application of supportive environments for people living with disability provide a theoretical and occupational lens through which participation, environmental modifications, and supports for noninstitutionalized adults aging with IDD can be examined. Oliver (2004) described the social model of disability as "a clear focus on the economic, environmental and cultural barriers encountered by people who are viewed by others as having some form of impairment—whether physical, sensory or intellectual" (p. 6). It is necessary, however, to underscore the distinction between impairment (e.g., physical, sensory, and intellectual) and the social positioning or construction of disability: "Disability is something imposed on top of our impairments by the way we are unnecessarily 
isolated and excluded from full participation in society" (Anastasiou \& Kauffman, 2013, p. 442). In other words, the construction of disability or IDD can inadvertently restrict participation in even the most mundane of daily life activities for people who occupy these identities; therefore, a critical examination of the intersections of person, environment, and disability is required for full consideration of the occupational needs of adults aging with IDD (Anastasiou \& Kauffman, 2013; Johnson et al., 2019; Law, 1991).

Law (1991) dissected notions of environments and client-centered practices that could move occupational therapy theory and practice forward using disability studies perspectives. More specifically, Law called attention to the need for practitioners to critically consider the environment, context of intervention for intervention planning, power, theoretical approaches, goal setting, and use of technologies in promoting supportive environments. Law's influence is evidenced through various occupational therapy practice models, for example, the Person-Environment-Occupation (PEO) Model (Law et al., 1996), which emphasizes how interactions between person and environment can positively or negatively influence occupational performance and participation, and the Person-Environment-Occupation-Performance (PEOP) Model, in which environment and occupational performance are reciprocal in nature (Christiansen et al., 2015; Law et al., 1996).

Social constructions of disability and their influence on the broader contexts of occupational therapy practice are complex and sometimes contradictory (Pollard \& Block, 2017). Scholars have described the social model of disability as an "outdated ideology" (Owens, 2015) that does not address disability as an attribute essential to a person's lived experience, does not account for different forms of disability experiences, and has not attended to institutionalized discrimination toward people with disabilities (Mirza et al., 2016; Terzi, 2004). Yet, a nuanced social view of disability is essential for responsive occupational therapy practice (Alcedo et al., 2017). Models and perspectives must reflect the contexts (e.g., sociopolitical and economic environments) of people with disabilities in their entirety. If occupational therapy practitioners do not do so, they risk misappropriating and perpetuating the marginalization of people with disabilities (Mirza et al., 2016). Therefore, we use the social model of disability, together with environmental and clientcentered perspectives, to ground, map, and categorize the analyses for this scoping review.

\section{Method}

We sought to identify literature specific to environmental modifications and supports, participation, and the social model of disability among the population of adults with IDD. Given the range of evidence and broad nature of the research question, we determined that a scoping review was the most appropriate methodology to review and map relevant literature. We followed the five-step process outlined by Arksey and O'Malley (2005) to ensure that the scoping review was standardized and comprehensive.

\section{Step 1}

We developed the following research question: What environmental modifications and supports are used to facilitate accessibility and occupational participation for people aging with IDD?

\section{Step 2}

One author (Selena E. Washington) received initial consultation from a university medical librarian with vast experience in conducting systematic and scoping reviews. The medical librarian completed a custom search of the literature using PubMed and Scopus. PubMed and Scopus are optimal tools in electronic research and enable a wide key word search of peer-reviewed literature in the fields of biomedicine, humanities, and occupational and social sciences (Falagas et al., 2008). The following Medical Subject Headings search terms and general terms were used: home or residential facilities or independent living or residential home, and middle age or aged, and activities of daily living, and intellectual disability, and/or developmental disabilities. We intentionally included the term activities of daily living 
(ADLs), which numerically yielded larger search results specifically related to participation. The search terms were filtered to include content in abstracts and in the English language. Reference lists of included studies and review articles identified in the initial search were screened to locate additional relevant studies.

\section{Step 3}

We used the following inclusion criteria for the studies: (1) The study was written in the English language; (2) occupational participation was based on environmental modification (e.g., physical space, technology, universal design, type of living setting) or on environmental and social supports (e.g., family members, caretakers, peer groups, health care professionals, policies, organizational infrastructure; World Health Organization, 2017); (3) type of disability was intellectual disability, developmental disability, or disability identified by IQ level (i.e., profound, severe, moderate, or mild); and (4) the study included participants age $35 \mathrm{yr}$ or older. No restriction was placed on the year of publication. Meta-analyses and systematic reviews were excluded.

\section{Step 4}

To calibrate the inclusion and exclusion criteria and to ensure consistency in title and abstract screening, the medical librarian and one reviewer (Washington) performed the initial search. Next, a second reviewer (Jesse M. Hollenbeck) independently verified the title and abstract screening for accuracy. No discrepancies were found. The literature was descriptively and independently charted by two authors (Washington and Hollenbeck). The extracted literature was organized through the use of an Excel spreadsheet and Google Drive according to the following predetermined fields: journal of publication, study design and description, theory and conceptual framework, intervention, assessment and outcome measures used, and study findings. The chosen articles $(N=11)$ were cross-checked for discrepancies. The authors and reviewers of this study were located within the United States and the United Kingdom.

\section{Step 5}

Two reviewers (Washington and Hollenbeck) independently undertook full-text screening, and no conflicts arose (Figure 1). They summarized the literature to identify major themes, gaps, and areas of interest for this scoping review. A thematic analysis was performed to identify and arrange salient themes on the basis of Law's (1991) descriptions of environmental supports and barriers. All three authors classified the outcome descriptions under the most relevant corresponding environmental modification and support concepts or domains to align with the theoretical lens, as discussed in the introduction (Table A.1 in the Appendix).

\section{Results}

The initial search yielded 298 publications. After the removal of 33 duplicates, 265 abstracts were reviewed against the full inclusion criteria. Eleven studies met the inclusion criteria for this scoping review (see Figure 1). Sample sizes ranged from 15 to 13,642 participants. The studies were organized under one or more environmental themes (Law, 1991), as noted in Table A.1: definition of need $(n=5)$, environmental risk and assessment $(n=1)$, environmental setting $(n=6)$, intervention and program planning $(n=7)$, use of technology $(n=1)$, and bureaucracy $(n=1)$. All studies targeted adults aging with IDD (age $\geq 18 \mathrm{yr}$ ). Studies primarily included participants who had an IQ in the mild to moderate range.

Nine of the studies were published in IDD-specific journals, and the other 2 studies were published in the Scandinavian Journal of Occupational Therapy and Health and Social Care in the Community. Seven studies used quantitative methods, and 4 studies used a qualitative approach. The studies explored the themes from Law's (1991) descriptions of environmental supports and were coded by two of the authors (Washington and Hollenbeck; see Table A.1). 
Figure 1. Flow diagram for inclusion and exclusion of peer-reviewed studies in the scoping review.

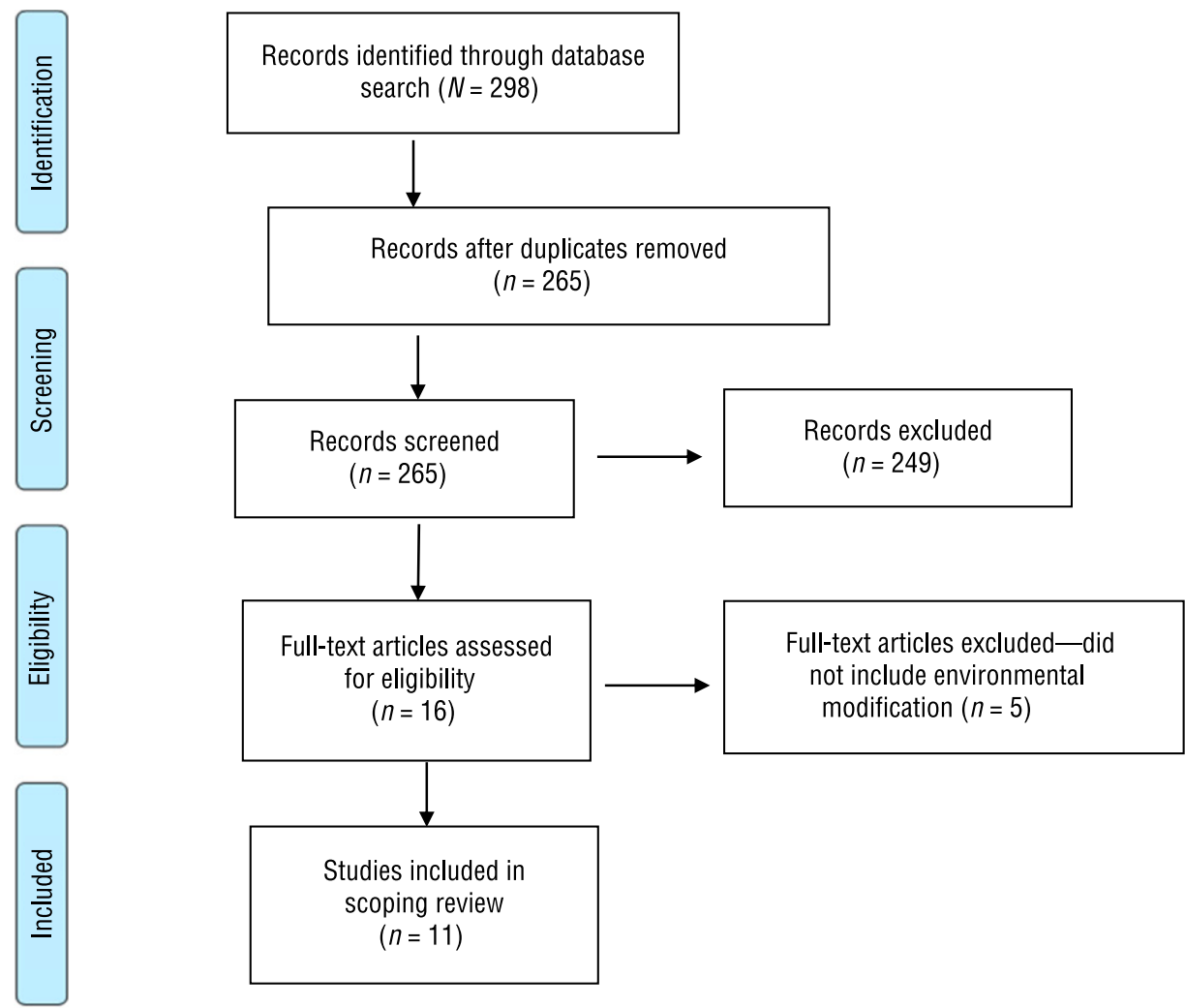

Note. Figure format from "Preferred Reporting Items for Systematic Reviews and Meta-Analyses: The PRISMA Statement," by D. Moher, A. Liberati, J. Tetzlaff, and D. G. Altman; PRISMA Group, 2009, PLoS Medicine, 6(7), e1000097. https://doi.org/10.1371/journal.pmed.1000097

\section{Definition of Need}

Five articles described the need for intervention on the basis of the rights of those with disabilities to engage in meaningful occupations with environmental supports (Law, 1991). Burke et al. (2019) used the person-environment fit model (Law et al., 1996) to guide the client and caregiver decision-making process and to narrow the area of need for services and supports; this model was used to identify the best fit for housing and vocational environments. Through this model, Burke et al. identified a lack of environmental supports (i.e., service delivery for viable housing and work options) as one of the key determinants of participation. Lifshitz et al. (2008) and McCausland et al. (2010) emphasized the need for advocacy for caregivers and occupational choice among adults aging with IDD. In both of these studies, the researchers concluded that continuous engagement in functional activity tasks and lifelong learning programs focused on instrumental activity of daily living (IADL) tasks (education and financial skills) are enhanced by environmental supports when paired with preventive and rehabilitative programming.

Rand and Malley (2017) and Davies et al. (2010) examined environments and supports from a macro-level approach. Davies et al. investigated global positioning system (GPS) technology to facilitate accessibility and choice when navigating public transportation routes in the United States. Rand and Malley strategically explored the individual, environmental, and related characteristics associated with quality of life for adults with IDD using publicly funded supports in England; their study produced a large outcome data repository to facilitate system- and organizational-level change in society. 


\section{Environmental Risk and Assessment}

The theme of environmental risk and assessment encompasses the assessment of dysfunction in occupational participation and the identification of meaningful environmental settings (Law, 1991). Shaw et al. (2011) used focusgroup and individual interviews $(N=15)$ with clients aging with IDD ( $M$ age $=46 \mathrm{yr}$ ). Themes addressed (1) living arrangements (in which there was a strong preference to live near but not with peers aging with IDD), (2) housing preferences (adults aging with IDD preferred not to live alone or in small groups, and living in close proximity to peers was optimal), (3) aging in place (living near other adults aging with IDD was viewed as supporting the right to age in place and provided the greatest likelihood that social supports and engagement in occupation would be maintained), and (4) transition from informal to formal housing and support services (effective planning was required to manage this transition through accessibility, safety, choice, and flexibility of the housing environment).

\section{Environmental Setting}

The theme of environmental setting describes how the type of environmental setting affects behavior and occupational choices (Law, 1991). Six of the included studies focused on community-based home or environmental settings as well as occupational choice and participation. Community-based home or environmental settings refers to a house, apartment, group home, or home in a peer community, with or without others (i.e., those with a disability), with community-based support (King et al., 2017). Community-based environments and supports assisted participants in remaining in their home, maintaining ADL skills, and sustaining relationships with family and friends (Ellison et al., 2011; King et al., 2017; Rand \& Malley, 2017; Robertson et al., 2001). King et al. (2017) noted greater ability as well as ADL and IADL participation among adults aging with IDD residing in community-based home settings; type of residence strongly correlated with occupational and physical participation (King et al., 2017; Robertson et al., 2001). However, the Lifshftz (2008) study did not demonstrate a direct correlation between ADL participation and type of residence among aging adults with IDD. Young (2006) specifically noted significant improvement in adaptive behavior (awareness of environmental contexts and social cues and norms), choice making, and adaptive skills among adults aging with IDD who transitioned from an institutionalized setting to a community-based home setting.

\section{Intervention and Program Planning}

Intervention and program planning studies aimed to enable client experiences in various environmental settings that incorporate multiple levels of complexity appropriate to a person's interest, skills, and goals (Law, 1991). Seven studies reported positive outcomes with regard to the PEO Model, community-based support, occupational participation and engagement, and technology resources. Statistically significant changes were noted regarding adaptive behavior and participation depending on the type of environmental (housing) setting (King et al., 2017; Robertson et al., 2001). This type of significant change benefits adults aging with IDD by enabling them to safely participate in functional tasks in a home- or community-based living environment and to maintain adaptive behavior (i.e., awareness of environmental contexts and social cues and norms; Bailey, 2017; Tse et al., 2018).

Talman et al.'s (2019) study highlighted barriers to participation that were correlated with the lack of environmental supports needed to provide the environmental modifications for occupational participation. Interventions in the included studies consisted of government support and in-home provider services to improve participation (Ellison et al., 2011), optimal residential placement to facilitate engagement and participation (King et al., 2017; Robertson et al., 2001), and key environmental supports to sustain participation (Burke et al., 2019; Davies et al., 2010; Lifshitz et al., 2008).

\section{Use of Technology}

Davies et al. (2010) investigated the use of GPS technology to facilitate accessibility and choice when navigating public transportation routes within the United States. Their study addressed environmental barriers of transportation (i.e., route 
complexity, unfamiliar destinations, and schedule complexity) through the use of technology. This study's findings demonstrated that a GPS-enabled portable device, which included visual prompts, resulted in $73 \%$ accuracy of reaching the intended landmark destination by the participants $(N=23)$. The application of GPS technology helped support people aging with IDD to successfully navigate public transit systems, providing evidence-based intervention strategies that specifically addressed transportation and environmental participation.

\section{Bureaucracy}

The theme of bureaucracy corresponds to structures and processes that are used to create environments that can constrain or facilitate participation among those in a society (Law, 1991). Rand and Malley (2017) explored bureaucracy with the Adult Social Care Outcomes Toolkit (ASCOT; Malley et al., 2012), a measure of safety, social participation, and occupation. Rand and Malley used the ASCOT assessment among adults with IDD who were noninstitutionalized to inform local and national policy measures regarding the individual and environmental service needs of this population. Their analysis included the following questions regarding home design aspects and location of the respondent's environment: a 5-point, interviewer-rated question regarding cleanliness and organization of the home; a question regarding the type of area in which the person lived (rural or urban); a 4-point, self-rated question regarding the design of the home; and a 4-point question regarding accessibility of the local area. Rand and Malley's study addressed how and what specific variables should be strategically targeted to improve utilization of publicly funded support to facilitate accessibility for people aging with IDD.

\section{Discussion}

In this scoping review, we explored a diverse range of peer-reviewed articles reporting on environmental modification and supports for participation in the disability and occupational therapy literature. Through the lens of the social model of disability and Law's (1991) perspectives, we outlined supports (social and physical) and environmental barriers.

\section{Assessment}

Three of the 11 studies included in this review specifically used assessments that measured environmental factors and participation, indicating a lack of published research with this target population. The included studies on assessment provide a client-centered approach to how environmental supports and settings affect participation (King et al., 2017; Rand \& Malley, 2017; Shaw et al., 2011); however, additional research on the qualitative and quantitative aspects of environmental factors that influence participation is warranted. As discussed previously, Hammel et al. (2015) outlined eight major categories of environmental factors that influence participation: built, natural, assistive technology, access to information and technology, transportation, social support and societal attitudes, economics, and systems and policies. Occupational therapy's transactive person-environment models (i.e., the PEO and PEOP Models; Christiansen et al., 2015; Law et al., 1996), along with the use of disability models, provide a unique conceptual lens to assess the aforementioned environmental factors and participation within this population.

\section{Intervention}

The number of adults aging with IDD to age $60 \mathrm{yr}$ and older in the United States is expected to increase by 1.4 million by 2030 as a result of the increased life expectancy of the baby boomer generation (Factor et al., 2012). This population is at risk for no longer being able to safely function in a home- or community-based living environment because of common health issues (e.g., aspiration, dehydration, constipation, seizure disorders), decreased adaptive behavior (i.e., awareness of environmental contexts, social cues, and norms), and early-onset, age-related health problems (e.g., physical decline or dementia; Bailey, 2017; Tse et al., 2018). The studies included in this scoping review outline how ongoing supports are essential to support aging in place for populations with IDD (Ellison et al., 2011; King et al., 2017; 
Robertson et al., 2001); expanded knowledge of strategic planning and community-based approaches is also critical (Heller, 2017; Talman et al., 2019). The use of disability models, examination of evidence-based interventions, and ongoing professional development in the occupational therapy profession are warranted to efficiently support this movement and to incorporate a client-centered approach.

Our findings complement Law's (1991) definition of the evolving occupational therapist; it is vital to evolve in the areas of responsiveness and client-centered care to address environmental concerns and solutions with this aging population. This scoping review addressed environmental modifications and supports and highlighted collective and individual outcomes within this population. For occupational therapy to move forward as a profession, it is important to develop additional evidence-based practice areas and to address the broader issue of disability rights and aging in place within this population. The social model of disability and Law and colleagues' (Law, 1991, 2002; Law et al., 1996) model guide our logic on how to generate environmental change and supports, identify barriers to participation, and define the relationship with regard to environmental factors.

\section{Advocacy}

A small body of literature provided evidence to support advocacy and policy work centering on social and environmental modifications that are essential for adults with IDD who are aging in place in the community (Burke et al., 2019; Davies et al., 2010; Lifshitz et al., 2008; McCausland et al., 2010; Rand \& Malley, 2017). The articles reviewed, which measured outcomes of safety, environmental demands for participation, health, and occupation (among others), can inform policies and practices aimed to improve the quality of life of this population. Therefore, it is imperative that assessments for people with unique needs consider the individual person who transacts through both the social and the physical environments. In other words, measurements must address the social and environmental factors that facilitate participation from micro to macro levels; doing so provides occupation-based evidence (or professional response) to inform planning and policy inherent in occupational therapy practice (Alcedo et al., 2017), not in addition to practice (Johnson et al., 2019).

\section{Limitations and Future Directions}

Potential limitations of this scoping review (Arksey \& O'Malley, 2005) include the exclusion of any relevant unpublished reports, master's theses and doctoral dissertations, and government publications on environmental modification and supports for participation among adults aging with IDD. We sought to examine peer-reviewed published articles to highlight how environmental supports and participation have been investigated in the disability and occupational therapy literature. The search strategy used for this study may have led to further limitations because of the omission of other potentially relevant databases. However, we believe that the risk that relevant studies were not identified by the search strategy is minimal because we consulted an experienced medical librarian.

The criterion that excluded studies written in any language other than English was also a limitation. In addition, the authors and reviewers were based in the United States; however, the review included studies from other countries. Given that the social model of disability and occupational therapy practice frameworks and models are constantly evolving (Kielhofner, 2005), findings from the included studies are applicable to this current population. However, future research clearly needs to develop relevant outcomes and evidence-based interventions for this population.

\section{Implications for Occupational Therapy Practice}

Findings from this scoping review have the following implications for occupational therapy practice in supporting further implementation of environmental modifications, supports, and participation for older adults with IDD:

- Practitioners and researchers should use empirical literature from this scoping review and other disability studies to guide innovative practice and client-centered interventions in community-based environments. 


\section{- Practitioners and researchers should engage in dialogue and advocacy to align with self-identified environmental}

needs of communities of those aging with disability.

- Researchers should prioritize validation and investigation of evidence-based interventions, targeting environmental and occupational barriers to participation identified in populations of adults aging with IDD.

Practitioners and researchers should use trend data identified by the National Institute on Disability, Independent Living, and Rehabilitation Research and the Administration for Community Living Aging and Disability Resource Centers to identify the most prevalent occupational and environmental issues.

\section{Conclusion}

This scoping review highlights collective and individual outcomes in the areas of advocacy, assessment, and intervention. Further research is needed within the scope of occupational therapy and disability studies that examines environmental factors and participation outcomes for this population. Future research and practice can be supported and expanded through collaboration with aging and disability communities and national organizations.

\section{References}

Alcedo, M. Á., Fontanil, Y., Solís, P., Pedrosa, I., \& Aguado, A. L. (2017). People with intellectual disability who are ageing: Perceived needs assessment. International Journal of Clinical Health and Psychology, 17(1), 38-45. https://doi.org/10.1016/j.ijchp.2016.07.002

American Association on Intellectual and Developmental Disabilities. (n.d). Definition of intellectual disability. http://aaidd.org/intellectual-disability/ definition\#.WdOb5GhSyUk

Anastasiou, D., \& Kauffman, J. M. (2013). The social model of disability: Dichotomy between impairment and disability. Journal of Medicine and Philosophy, 38, 441-459. https://doi.org/10.1093/jmp/jht026

Arksey, H., \& O'Malley, L. (2005). Scoping studies: Towards a methodological framework. International Journal of Social Research Methodology, 8, $19-32$. https://doi.org/10.1080/1364557032000119616

Bailey, R. (2017, February 6). The fatal four: Major health issues that impact individuals with intellectual and developmental disabilities. http://www.ancor.org/ resources/publications/links/fatal-four-major-health-issues-impact-individuals-intellectual-and

*Burke, M. M., Lee, C. E., Hall, S. A., \& Rossetti, Z. (2019). Understanding decision making among individuals with intellectual and developmental disabilities (IDD) and their siblings. Intellectual and Developmental Disabilities, 57, 26-41. https://doi.org/10.1352/1934-9556-57.1.26

Christiansen, C., Baum, C. M., \& Bass, J. D. (2015). Occupational therapy: Performance, participation, and well-being (4th ed.). Slack.

*Davies, D. K., Stock, S. E., Holloway, S., \& Wehmeyer, M. L. (2010). Evaluating a GPS-based transportation device to support independent bus travel by people with intellectual disability. Intellectual and Developmental Disabilities, 48, 454-463. https://doi.org/10.1352/1934-9556-48.6.454

*Ellison, C., White, A., \& Chapman, L. (2011). Avoiding institutional outcomes for older adults living with disability: The use of community-based aged care supports. Journal of Intellectual and Developmental Disability, 36, 175-183. https://doi.org/10.3109/13668250.2011.597377

Factor, A., Heller, T., \& Janicki, M. (2012, March 15). Bridging the aging and developmental disabilities service networks: Challenges and best practices. https://www.aarp.org/content/dam/aarp/livable-communities/old-learn/health/Bridging-the-Aging-and-Developmental-Disabilities-Service-Networks-

Challenges-and-Best-Practices-AARP.pdf

Falagas, M. E., Pitsouni, E. I., Malietzis, G. A., \& Pappas, G. (2008). Comparison of PubMed, Scopus, Web of Science, and Google Scholar: Strengths and weaknesses. FASEB Journal, 22, 338-342. https://doi.org/10.1096/fj.07-9492LSF

Hammel, J., Magasi, S., Heinemann, A., Gray, D. B., Stark, S., Kisala, P., .. Hahn, E. A. (2015). Environmental barriers and supports to everyday participation: A qualitative insider perspective from people with disabilities. Archives of Physical Medicine and Rehabilitation, 96, 578-588. https://doi.org/10.1016/j. apmr.2014.12.008

Heller, T. (2017, October 25). Service and support needs of adults aging with intellectual/developmental disabilities. Testimony to the U.S. Senate Committee on Aging. Working and aging with disabilities: From school to retirement. https://www.aging.senate.gov/imo/media/doc/SCA_Heller_10_25_17.pdf

Hole, R. D., Stainton, T., \& Wilson, L. (2013). Ageing adults with intellectual disabilities: Self-advocates' and family members' perspectives about the future. Australian Social Work, 66, 571-589. https://doi.org/10.1080/0312407X.2012.689307

Hutchings, B. L., Olsen, R. V., \& Moulton, H. J. (2008). Environmental evaluations and modifications to support aging at home with a developmental disability. Journal of Housing for the Elderly, 22, 286-310. https://doi.org/10.1080/02763890802458445

Johnson, K. R., \& Bagatell, N. (2017). Beyond custodial care: Mediating choice and participation for adults with intellectual disabilities. Journal of Occupational Science, 24, 546-560. https://doi.org/10.1080/14427591.2017.1363078

Johnson, K. R., Blaskowitz, M., \& Mahoney, W. (2019). Occupational therapy practice with adults with intellectual disability: What more can we do? Open Journal of Occupational Therapy, 7, 1-6. https://doi.org/10.15453/2168-6408.1573

*Indicates studies included in the scoping review. 
Kielhofner, G. (2005). Rethinking disability and what to do about it: Disability studies and its implications for occupational therapy. American Journal of Occupational Therapy, 59, 487-496. https://doi.org/10.5014/ajot.59.5.487

*King, E., Okodogbe, T., Burke, E., McCarron, M., McCallion, P., \& O’Donovan, M. A. (2017). Activities of daily living and transition to community living for adults with intellectual disabilities. Scandinavian Journal of Occupational Therapy, 24, 357-365. https://doi.org/10.1080/11038128.2016.1227369

Law, M. (1991). The environment: A focus for occupational therapy. Canadian Journal of Occupational Therapy, 58, 171-180. https://doi.org/10.1177/ 000841749105800404

Law, M. (2002). Participation in the occupations of everyday life. American Journal of Occupational Therapy, 56, 640-649. https://doi.org/10.5014/ajot.56.6. 640

Law, M., Cooper, B., Strong, S., Stewart, D., Rigby, P., \& Letts, L. (1996). The Person-Environment-Occupation Model: A transactive approach to occupational performance. Canadian Journal of Occupational Therapy, 63, 9-23. https://doi.org/10.1177/000841749606300103

*Lifshitz, H., Merrick, J., \& Morad, M. (2008). Health status and ADL functioning of older persons with intellectual disability: Community residence versus residential care centers. Research in Developmental Disabilities, 29, 301-315. https://doi.org/10.1016/j.ridd.2007.06.005

Malley, J. N., Towers, A.-M., Netten, A. P., Brazier, J. E., Forder, J. E., \& Flynn, T. (2012). An assessment of the construct validity of the ASCOT measure of social care-related quality of life with older people. Health and Quality of Life Outcomes, 10, 21. https://doi.org/10.1186/1477-7525-10-21

*McCausland, D., Guerin, S., Tyrrell, J., Donohoe, C., O’Donoghue, I., \& Dodd, P. (2010). Self-reported needs among older persons with intellectual disabilities in an Irish community-based service. Research in Developmental Disabilities, 31, 381-387. https://doi.org/10.1016/j.ridd.2009.10.005

Mirza, M., Magasi, S., \& Hammel, J. (2016). Soul searching occupations: Critical reflections on occupational therapy's commitment to social justice, disability rights, and participation. In P. Block, D. Kasnitz, A. Nishida, \& N. Pollard (Eds.), Occupying disability: Critical approaches to community, justice, and decolonizing disability (pp. 159-174). Springer. https://doi.org/10.1007/978-94-017-9984-3_11

Miskimmin, C., Shooshtari, S., Menec, V., Duncan, K. A., Martin, T., \& Stoesz, B. M. (2019). Age-friendly communities for older persons with intellectual disabilities. Quality in Ageing and Older Adults, 20, 206-218. https://doi.org/10.1108/QAOA-11-2018-0058

Oliver, M. (2004). The Social Model in action: If I had a hammer. In C. B. Barnes \& G. Mercer (Eds.), Implementing the social model of disability: Theory and research (pp. 18-31). Disability Press. https://disability-studies.leeds.ac.uk/wp-content/uploads/sites/40/library/Barnes-implementing-the-socialmodel-chapter-2.pdf

Owens, J. (2015). Exploring the critiques of the social model of disability: The transformative possibility of Arendt's notion of power. Sociology of Health and Illness, 37, 385-403. https://doi.org/10.1111/1467-9566.12199

Pollard, N., \& Block, P. (2017). Who occupies disability? Cadernos Brasileiros de Terapia Ocupacional, 25, 417-426. https://doi.org/10.4322/0104-4931. ctoEN18252

*Rand, S., \& Malley, J. (2017). The factors associated with care-related quality of life of adults with intellectual disabilities in England: Implications for policy and practice. Health and Social Care in the Community, 25, 1607-1619. https://doi.org/10.1111/hsc.12354

${ }^{*}$ Robertson, J., Emerson, E., Hatton, C., Gregory, N., Kessissoglou, S., Hallam, A., \& Walsh, P. N. (2001). Environmental opportunities and supports for exercising self-determination in community-based residential settings. Research in Developmental Disabilities, 22, 487-502. https://doi.org/10.1016/ S0891-4222(01)00085-3

Schalock, R. L. (2004). The concept of quality of life: What we know and do not know. Journal of Intellectual Disability Research, 48, 203-216. https://doi.org/ 10.1111/j.1365-2788.2003.00558.x

Schepens, H. R. M. M., Van Puyenbroeck, J., \& Maes, B. (2019). How to improve the quality of life of elderly people with intellectual disability: A systematic literature review of support strategies. Journal of Applied Research in Intellectual Disabilities, 32, 483-521. https://doi.org/10.1111/jar.12559

*Shaw, K., Cartwright, C., \& Craig, J. (2011). The housing and support needs of people with an intellectual disability into older age. Journal of Intellectual Disability Research, 55, 895-903. https://doi.org/10.1111/j.1365-2788.2011.01449.x

Spassiani, N. A., Meisner, B. A., Abou Chacra, M. S., Heller, T., \& Hammel, J. (2019). What is and isn't working: Factors involved in sustaining communitybased health and participation initiatives for people ageing with intellectual and developmental disabilities. Journal of Applied Research in Intellectual Disabilities, 32, 1465-1477. https://doi.org/10.1111/jar.12640

*Talman, L., Wilder, J., Stier, J., \& Gustafsson, C. (2019). Staff members and managers' views of the conditions for the participation of adults with profound intellectual and multiple disabilities. Journal of Applied Research in Intellectual Disabilities, 32, 143-151. https://doi.org/10.1111/jar.12516

Terzi, L. (2004). The social model of disability: A philosophical critique. Journal of Applied Philosophy, 21, 141-157. https://doi.org/10.1111/j.0264-3758. 2004.00269.x

Tse, M. M., Kwan, R. Y., \& Lau, J. L. (2018). Ageing in individuals with intellectual disability: Issues and concerns in Hong Kong. Hong Kong Medical Journal, 24, 68-72. https://doi.org/10.12809/hkmj166302

World Health Organization. (2017). International classification of functioning, disability and health. https://www.who.int/standards/classifications/internationalclassification-of-functioning-disability-and-health

*Young, L. (2006). Community and cluster centre residential services for adults with intellectual disability: Long-term results from an Australian-matched sample. Journal of Intellectual Disability Research, 50, 419-431. https://doi.org/10.1111/j.1365-2788.2006.00788.x 
Selena E. Washington, PhD, OTR/L, is Assistant Professor, Department of Occupational Science and Occupational Therapy, Saint Louis University, St. Louis, MO; selena.washington@health.slu.edu

Khalilah R. Johnson, PhD, OTR/L, is Assistant Professor, Division of Occupational Science and Occupational Therapy, University of North Carolina at Chapel Hill.

Jesse M. Hollenbeck, MPH, is Graduate Assistant, College for Public Health and Social Justice, Saint Louis University, St. Louis, MO.

\section{Acknowledgments}

We thank Susan Stark for her professional mentorship and guidance and Donghua Tao for her efforts and contributions to this review. 


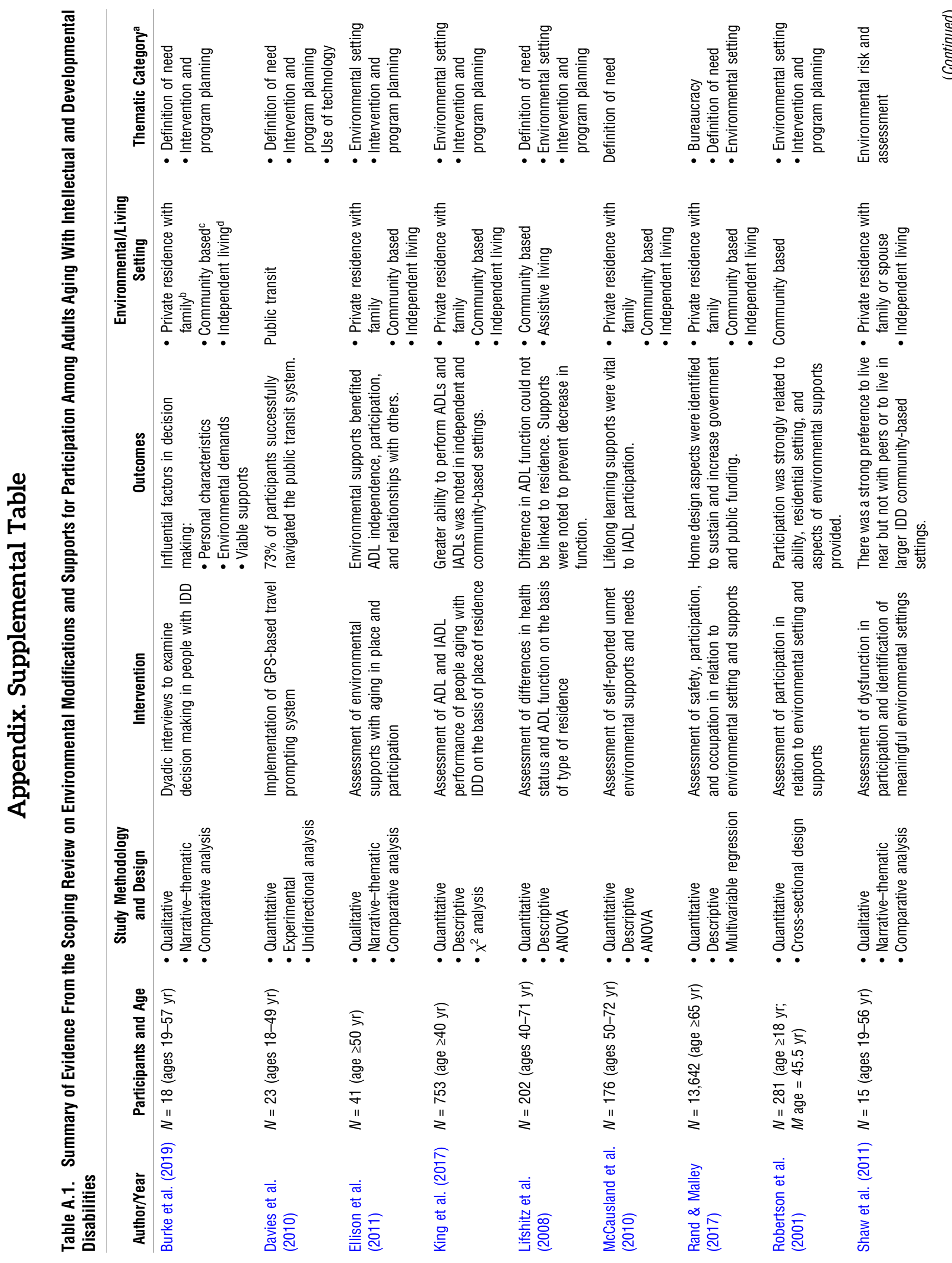




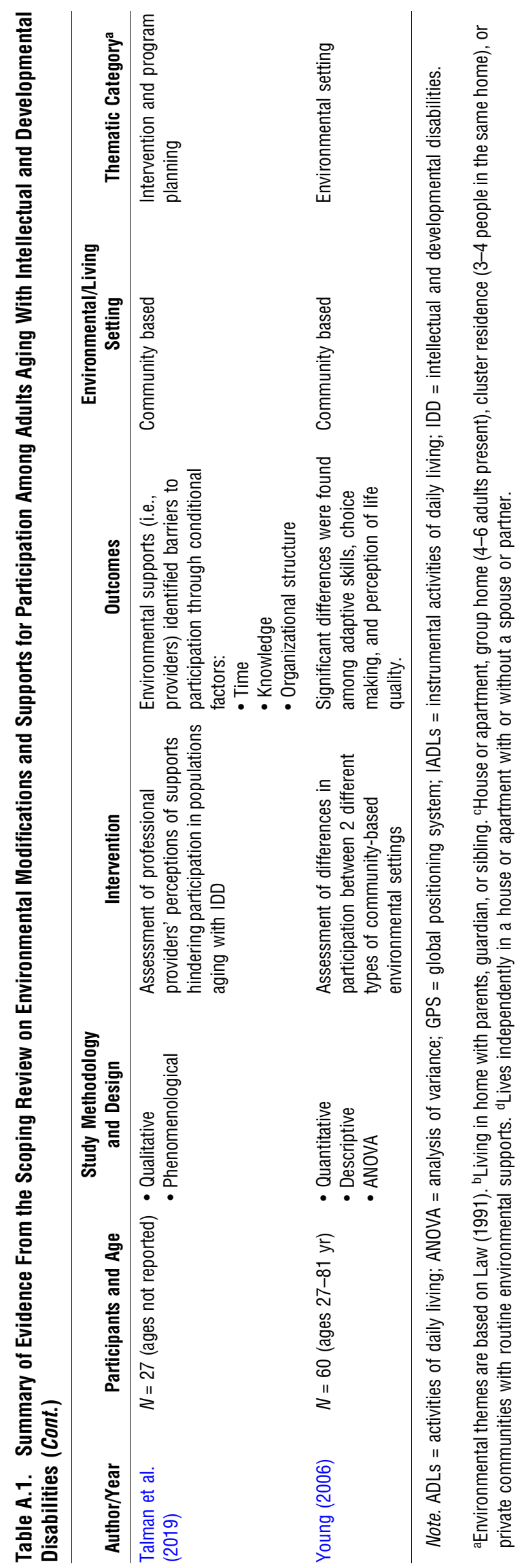

